\section{cambridge.org/par}

\section{Special Issue Review}

Cite this article: Molyneux $\mathrm{DH}$, Dean $\mathrm{L}$, Adekeye O, Stothard JR, Theobald S (2018). The changing global landscape of health and disease: addressing challenges and opportunities for sustaining progress towards control and elimination of neglected tropical diseases (NTDs). Parasitology 145, 1647-1654. https://doi.org/10.1017/S0031182018000069

Received: 20 October 2017 Revised: 18 December 2017 Accepted: 4 January 2018

First published online: 16 March 2018

\section{Key words:}

disability; neglected tropical diseases; preventive chemotherapy; sustainable development goals; universal health coverage

Author for correspondence:

David H. Molyneux, E-mail: david.molyneux@ Istmed.ac.uk

\title{
The changing global landscape of health and disease: addressing challenges and opportunities for sustaining progress towards control and elimination of neglected tropical diseases (NTDs)
}

David H. Molyneux, Laura Dean, Oluwatosin Adekeye, J. Russell Stothard and Sally Theobald

Department of Parasitology, Liverpool School of Tropical Medicine, Pembroke Place, Liverpool L3 5QA, UK

\section{Abstract}

The drive to control neglected tropical diseases (NTDs) has had many successes but to reach defined targets new approaches are required. Over the last decade, NTD control programmes have benefitted from increased resources, and from effective partnerships and long-term pharmaceutical donations. Although the NTD agenda is broader than those diseases of parasitic aetiology there has been a massive up-scaling of the delivery of medicines to some billion people annually. Recipients are often the poorest, with the aspiration that NTD programmes are key to universal health coverage as reflected within the 2030 United Nations sustainable development goals (SDGs). To reach elimination targets, the community will need to adapt global events and changing policy environments to ensure programmes are responsive and can sustain progress towards NTD targets. Innovative thinking embedded within regional and national health systems is needed. Policy makers, managers and frontline health workers are the mediators between challenge and change at global and local levels. This paper attempts to address the challenges to end the chronic pandemic of NTDs and achieve the SDG targets. It concludes with a conceptual framework that illustrates the interactions between these key challenges and opportunities and emphasizes the health system as a critical mediator.

\section{Introduction}

Neglected tropical diseases (NTDs) are some of the most common infections of poor people in impoverished communities not only in the poorest low- and middle-income countries (LMICs) but also in the richer G20 countries (Hotez, 2017). Several of these diseases (lymphatic filariasis, onchocerciasis, soil-transmitted helminths, schistosomiasis and trachoma) are amenable to preventive chemotherapy (PC). With progress in the delivery of treatments, there are opportunities to transition from control to elimination of these diseases, or to verify the absence of further transmission as outlined in the World Health Organization (WHO) 2020 NTD roadmap (WHO, 2012). As we approach elimination targets for PC NTDs in many settings, programmes face numerous challenges that can be grouped into core categories - biological and technological, political and social, and environmental (Bockarie et al. 2013; Webster et al. 2014). This paper explores how the health system can become a mediator between such challenges at the local level and those at the national/international level. Specifically, we argue how responsive and resilient health systems are essential to reach the aspiration of the NTD 'endgame' as articulated in the sustainable development goals (SDGs).

\section{Global shocks: a disrupting factor for NTD control}

Since 2007, a series of global events that span the social, environmental and political, present a challenging set of circumstances against which health systems, encompassing NTD programmes, have been required to adapt such as the global financial crisis, increased conflict, drought and the emergence of terror organizations. These events have induced almost unprecedented migration, creating refugee and displaced persons crises and stressing already weak and fragile health systems. The impact of such crises was exemplified during the 2014 Ebola (EVD) epidemic in Liberia, Sierra Leone and Guinea. EVD originated in remote and difficult to access communities, with complex health-seeking pathways, a weak and poorly resourced health system, and limited surveillance capacity. In these contexts, the health system faced an unprecedented challenge given the high case mortality rates and wide-reaching psychosocial impacts. Diversion of resources to control this EVD epidemic caused a necessary acute polarization of global and national health systems and focussed attention away from other chronic problems, such as NTDs. This presented an impediment to NTD control in the affected regions, specifically in Sierra Leone where significant progress had previously been made (Hodges et al. 2011; Pose and Rabinowitch, 2014), as well as in Guinea and 
Liberia where successes in onchocerciasis control has led to the emergence of NTD programmes supported initially by Non-Governmental Development Organizations (NGDOs) (Bogus et al. 2016; Thomas et al. 2017). Thus, global and national shocks frequently become a significant disrupting factor for NTD control, and create a challenge for the ability of health systems to respond appropriately to changing population needs, whilst also supporting the 'everyday resilience' that allows for the ongoing functioning of routine activity, such as immunization and NTD programme delivery (Gilson et al. 2017).

\section{Changing global dynamics: a need for better control of vectors}

Biological shifts that allow for the rapid emergence and potential spread of vector-borne viruses, are exemplars of another type of challenge facing the global health community. The emergence of Zika virus in the Americas in 2016, has emphasized the need for better surveillance systems and improved awareness of the risks of vector-borne viruses. The expansion of the Aedes populations also poses significant threats to the control of dengue and Chikungunya, as these Aedes transmitted viruses are expanding their range, for example, into the Gulf Coast States of the USA. Yellow fever epidemics have also occurred more recently in central Africa and Brazil and as a result, the WHO have initiated an Eliminating Yellow Fever Epidemic (EYE) strategy with the aim to eliminate such epidemics (WHO, 2017a). Despite such changing biological dynamics, there have been many historical successes in vector control dating back to 1904 including the control of malaria and yellow fever in Panama; malaria in Brazil (with the "eradication" of Anopheles gambiae); onchocerciasis in West Africa; control of dengue in Singapore and Cuba; some initial impact of vector control on Triatome transmission of Chagas' Disease in the Southern Cone of the Americas; filariasis control in the Solomon Islands because of indoor residual spraying (IRS) against malaria vectors (Webber, 1979); and success in filariasis programmes through vector control using bed-nets in Nigeria, The Gambia and Zambia (Blackburn et al. 2006; Eigege et al. 2013; Rebollo et al. 2015; Nsakashalo-Senkwe et al. 2017). Perhaps the most notable impact of vector control, however, has been the reduction in malaria morbidity and mortality attributed to long-lasting insecticide-impregnated bed-nets (LLIN) in sub-Saharan Africa. An estimated $68 \%$ of the decline in malaria prevalence was attributed to vector control (including IRS) between 2000 and 2015 (Bhatt et al. 2015; WHO, 2016).

Despite these historic successes, however, and the clear impact that vector control can have in addressing many global health challenges, vector control has been side-lined in attempts to control and eliminate many NTDs. Many elimination targets focus on a PC strategy with limited recognition that infections are acquired by insect bites or contact with freshwater snail habitats. In elimination programmes, transmission control is the key and too often the opportunity to address transmission has been underrecognized. The concept of mass drug administration (MDA) in lymphatic filariasis and onchocerciasis is designed to reduce the circulating populations of microfilariae to reduce transmission without vector control; however, the inclusion of vector control initiatives would greatly enhance the progression towards the elimination targets (Hollingsworth et al. 2015). Progress in control of vectors of other parasitic infections, such as human African trypanosomiasis, i.e. Glossina spp. (Tirados et al. 2015), Chagas' Disease (Dias et al. 2002) and visceral leishmaniasis, phlebotomine sandflies (Coleman et al. 2015), are critical to the achievement of elimination targets.

While there is no doubt that vector control should be a key pillar of NTD interventions, the challenges of emerging insecticide resistance in Anopheles populations in Africa, pose a threat to the continued impact of bed nets and LLINs (Hemingway et al. 2016). Furthermore, there has been limited recognition of the role of vectors and the need to increase the focus on transmission control. For example, whilst the WHO roadmap emphasizes the need for new drugs and diagnostics, as well as revitalized tools for monitoring and evaluation, demand for additional vector control tools and approaches has been noticeably absent (WHO, 2012). However, in its third NTD report WHO did highlight the additional cost of vector control which would be required to meet some of the control targets for infections such as dengue (this report was issued prior to the emergence and spread of Zika virus). More recently, WHO has responded to the increased threat of vector-borne infections and published a draft Global Vector Control Response 2017-2030 (WHO, 2017b). This document highlights the contributions which vector control has historically made to public health and links vector control interventions to the SDGs.

\section{Environmental shifts}

Rapid environmental change resulting in a less stable and unpredictable climate has also impacted on NTD epidemiology. These changes include: deforestation, mineral extraction and exploitation; habitat destruction and desertification; changes in water resource availability; severe climate change driving major flood events and landslides (e.g. China, Bangladesh, Sierra Leone); more frequent droughts (e.g. Africa) with consequences for food security; and associated threats to livestock husbandry and animal well-being as traditional agricultural practices create local conflicts over land and water resources. Environmental shifts coupled with changing global patterns and human-animal interactions reinforce the need for a 'one health' approach. For example, the World Health Assembly (WHA) resolution of 2013 on NTDs emphasized that the control of zoonotic diseases as a core NTD strategy whilst the research priorities were identified to address the major zoonotic diseases - rabies, echinococcosis, taeniasis and neurocysticercosis in the WHO NTD portfolio (WHO, 2015a).

The neglected within the neglected: innovative approaches required for impact

\section{Thinking beyond preventive chemotherapy diseases}

To date, NTD efforts have focused on the up-scaling of PC by MDA, fuelled by large-scale drug donations. This has posed challenges for the management of other NTDs, which require intensive disease management (IDM) such as the trypanosomiases, Buruli ulcer, leprosy and the leishmaniases which have not been attributed the same level of resources despite their capacity to be fatal if untreated and the donation of curative drugs. Such IDM diseases can be more focal in their geographical distributions and thus present a need for more nuanced and directive interventions and a need for greater clinical expertise for diagnosis and treatment. There are, however, serious deficits in the therapeutic armamentarium for IDM diseases, which have led to continued investment in and advocacy for, this critical cause by the Drugs for Neglected Diseases initiative (DNDi) (www.dndi. org) and significant progress has been made in reducing the burden of human African trypanosomiasis and visceral leishmaniasis (WHO, 2016; Molyneux et al. 2017). However, those who remain with chronic clinical, and often irreversible symptoms because of such diseases, urgently require follow-up action and support. Furthermore, the foundation of the NTD programme through 'vertically' implemented MDA (which frequently by-passes health systems infrastructure) further compounds this challenge, as staff 
working within routine health services often lack clinical knowledge and capacities to be able to diagnose and manage such conditions. In these situations, a health systems as usual response is insufficient to address complex chronic conditions and innovative approaches are needed to reach an often diverse sub-set of a population who are disproportionately affected by significantly disabling and potentially life-threatening NTDs.

\section{A need for alternative strategies to enhance preventive chemotherapy mass drug administration}

In West and Central Africa, occurrence of lymphatic filariasis and onchocerciasis in areas of Loa loa endemicity, pose a significant challenge to elimination goals because of the severe adverse events (SAEs) associated with the impact of ivermectin on individuals with high parasitaemias of Loa microfilaria $\left(>30000 \mathrm{ml}^{-1}\right)$, exacerbating risk of encephalopathy (Gardon et al. 1997; Boussinesq, 2006). Over nearly two decades, extensive studies have concentrated research approaches on seeking to understand the pathology of SAEs as well as searching for strategies to identify individuals at risk, and where lymphatic filariasis is co-endemic, to define alternative strategies. Such strategies have included the use of twice-a-year albendazole, supplemented with vector control (Pion et al. 2017). There has, however, been limited consideration of alternative approaches by investing in testing the potential of reducing transmission by the vector Chrysops; if the transmission of $L$. loa in areas of high Loa endemicity could be reduced, the numbers of individuals with high to moderate parasitaemias would decline, hence the risk of SAEs would be reduced as high adult worm loads (driven by high levels of transmission) would also reduce. The neglect of the Chrysops dimension in considering the 'Loa problem' is reflected in the fact that the first review of Chrysops biology for over 50 years was published in 2017 (Kelly-Hope et al. 2017). This recent review includes suggestions for implementing vector control, e.g. the use of 'tiny targets', which are widely deployed for Glossina control (Tirados et al. 2015) and could have significant potential for reducing Chrysops populations. Thus, alternative strategies that prioritize vector control, as well as new drug distribution mechanisms, are likely to be essential in addressing more complex NTD scenarios.

\section{Thinking beyond the parasite: addressing long-term manifestations of NTDs}

There has been considerable debate about the burden attributed to NTDs by the global burden of disease (GBD) studies (Murray et al. 2012) and the discrepancy between earlier estimates of burden (Hotez et al. 2014). The estimates by the GBD 2010 attributed some 27 million disability-adjusted life years (DALYs) to NTDS whilst a study in 2014 (which included the 17 WHO NTDs as well as other NTD conditions) attributed 47.9 million DALYs (Hotez et al. 2014). Calculations of the mortality associated with NTDs according to the GBD gives an annual mortality of 150000 , a figure which has been challenged (Molyneux et al. 2017) as deaths from trematode-induced cancers, epilepsy (caused by neurocysticercosis) rabies, and snake bite were not included. The WHO figure of schistosomiasis associated deaths in Africa has also been estimated to be 20 times higher than GBD estimates, as none of the conditions have been correctly attributed to the NTD group of diseases but included as injuries, cancers and neurological conditions.

These discrepancies could reflect the impact of interventions over the past decade but the attribution of the disability weights and overall mortality of NTDs remains controversial, as disability weights and prevalence are key drivers in the calculations of DALYs. Gross underestimates of the prevalence of cutaneous leishmaniasis (Bailey et al. 2017) exemplifies this problem where residual scarring of a cured condition is not included, despite the social and mental health sequelae caused by the condition (Bailey et al. 2017). The mental health co-morbidities have recently been identified as being of increased significance as a major and chronic morbidity (Litt et al. 2012) in many of the NTDs. The mental health burden estimated for lymphatic filariasis is two to three times higher than those estimated by the GBD study for this condition using highly conservative figures. In addition, the mental health burden which caregivers suffer in the care of chronic NTD patients has only been assessed in filariasis (Ton et al. 2015). In the study of DALYs averted for ten major NTDs (de Vlas et al. 2016), impaired cognitive development due to soil-transmitted helminths and schistosomiasis infection, mental health morbidity, discrimination and stigma due to disfigurement, social exclusion resulting in poor marital prospects, the impact of catastrophic health expenditures and economic impact of reduced ability to work, were not included in the DALYs averted calculations exemplifying the need to adopt a more holistic appreciation of the overall NTD burden (Litt et al. 2012). Conversely, the apparent absence of consideration of NTDs by the mental health community as a cause of and a contribution to global mental health morbidity, and the need to address the patient and caregiver needs to give the existence of so many chronic NTD patients, should bring NTD and mental health communities together (Ferrari et al. 2013). Mental health is already projected to be the largest cause of Global Disease Burden by 2030, without consideration of the contribution of NTDs. Including NTDs within these mental health projections are important and will further elevate the serious comparative lack of recognition for both sets of diseases.

Furthermore, where completed, measures of disease associated morbidity (including mental health and disability) are frequently quantitatively defined. Given the complexity and multifaceted manifestations of NTDs at both the individual and community level, research that allows for more nuanced understandings from the perspectives of affected individuals is important (Reidpath et al. 2011). Historically, the limited acknowledgement of the lifelong morbidity associated with such diseases (Mieras et al. 2016), has led to the highly medicalized focus of morbidity measurement and programme implementation, e.g. the need to restore sight or reduce physical impairment related to NTDs such as lymphatic filariasis. Currently, the shift within international NTD policy and programming to recognize the need for a holistic approach to the control and management of NTDs through the presentation of strategies for disease management, disability and inclusion (DMDI) (Mieras et al. 2016) represents the opportunity to develop morbidity measures that are responsive to the needs and experiences of affected populations, and how they vary by differing axes of inequality, such as age, gender, stage of disease, experience of disability and whether living with one or several NTDs. Only when the complexities of individual and household realities are understood and measured in relation to NTDs are the needs of affected populations likely to be prioritized and addressed.

\section{Harnessing partnerships and political commitments}

\section{The importance of partnerships}

There are opportunities for holistic multi-sectoral action to support robust, resilient and responsive health systems responses to mediate challenges and sustain NTD control. Notably, NTD partnerships require financial resources and management time and the overall financial envelope has not increased; in 2010, the proportion of Official Development Assistance (ODA) committed to NTD programmes was $0.6 \%$ (Liese and Schubert, 2009). Funds 
over the last 7 years have increased despite the higher profile for NTDs (Liese et al. 2014). However, the diversity of interests and the numbers of partners involved has expanded significantly and this reflects the increasing recognition of the importance of these diseases. In addition, there is an ever more complex partner and donor landscape both in terms of implementation of diseasespecific approaches and integrated country programmes. A recent estimate provided by the infontd website (www.infontd.org) has identified over 2609 projects on NTDs in 113 countries involving 73 different partners.

Disease-specific partnerships have evolved and expanded in the last decade to provide focal points for endemic country partners, ministries of health, researchers and donors including WHO and the NGDO community (Liese et al. 2010). Such partnerships have an important advocacy role and enhance communication, networking, and technological advancements. The expansion of NGDO interest in NTDs has seen the development of the NNN-NGDO/NTD/Network over recent years (www.ntd-ngonetwork.org), as well as the Coalition for Operational Research COR-NTD (www.ntdsupport.org/cor-ntd) which identifies and supports operational research priorities. Research partnerships that span all areas of the translational research spectrum are also of critical importance in increasing the adaptability of NTD programmes in relation to emergent challenges.

Partnerships, however, must be based on parity of esteem of partners, transparency of management, the capacity to recognize sensitivities and manage them, regular communication and adequate resourcing. Most importantly, they should prioritize equity between colleagues in the global north and global south and be responsive to the needs and priorities of health systems and populations in LMICs who face the largest burden of NTDs. The significance of cross-cutting issues in the control of NTDs such as links to the WASH sector (WHO, 2015b), education, environment, agriculture and livestock, are important prerequisites to meeting the United Nations SDGs (Bangert et al. 2017). Partnerships that include expertise across and within such sectors and prioritize multi-disciplinary and multi-sectoral action are of critical importance if we are to reach the NTD 'endgame'. Below we illustrate the range of strategic partnerships needed across the translational research continuum: T1 (basic research), T2 (human/clinical research), T3 (evidence into practice) and T4 (practice to policy).

\section{Partnerships for drug development (T1-T2)}

The anti-Wolbachia (A.WOL) consortium reflects the importance of building a partnership for drug development using the comparative advantage of academic institutions and industry partners. The partnership focuses on the development of an effective macrofilaricide against adult filaria worms based on the efficacy of antibiotics against Wolbachia endosymbionts of Onchocerca and Wuchereria (www.awol.lstmed.ac.uk). Having demonstrated the efficacy of doxycycline as a macrofilaricide, A.WOL have promising candidate alternatives which can shorten the duration of treatment needed to secure adult worm death or permanent sterilization of infection. Such alternatives include high-dose rifampicin (Aljayyoussi et al. 2017) and a new entity TylAMac ${ }^{\mathrm{TM}}$ (Turner et al. 2015).

\section{Partnership for innovation and co-implementation across the disease programmes (T1-T4)}

The Integrated Vector Control Consortium (IVCC) (www.ivcc. com) is an example of the development of novel new vector control products which will ensure that vector control successes can be maintained in the future. IVCC is the only partnership involving academia, WHO, and the pesticide industry, focused on seeking alternative products for vector control which contrast to the several partnerships formed for addressing new drugs and diagnostics such as DNDi (www.dndi.org), the Medicine for Malaria Venture (MMV) (www.mmv.org), and the Foundation for Innovative New Diagnostics (FIND) (www.finddx.org). An irony upon which it is worth reflecting, is that a single new chemical entity effective against vectors, as synthetic pyrethroids have been over several decades, will impact on all vectors of infectious agents. Hence, it could be argued that proportionately more resources should be devoted to the search for new vector control products given the extent of pyrethroid resistance (Hemingway et al. 2016). Targeting vectors will have the most effective impact on transmission control and contribute proportionately more to elimination. Hence a 'pan' vector control product would have a potentially greater impact than a drug for one specific condition.

Building on the successes of vector control, the operationalization of the strategic partnership between the lymphatic filariasis and the malaria programme in Nigeria has shown the benefit of co-implementation through the combined distribution of LLIN. The key success of this programme was the endorsement in the policy of a synergistic approach of distribution of MDA and LLIN (Dean et al. 2016).

\section{Partnerships for Health Systems Strengthening (T3-T4)}

COUNTDOWN is a multi-disciplinary health systems implementation research programme which brings together NTD programmes and research institutes to address country priorities and challenges for strengthening NTD programme implementation. COUNTDOWN research takes place in Ghana, Cameroon, Nigeria and Liberia. For example, in Nigeria, following a situational analysis, key challenges were identified in four broad areas including: (i) co-ordination and collaboration; (ii) financial and non-financial resource mobilization; (iii) long-term multicontext community engagement; and (iv) human resources management and motivation (www.countdownonntds.org). Following identification of these challenges, COUNTDOWN worked with the Federal Ministry of Health to develop a holistic implementation research package and will work with the Federal and State (Ogun and Kaduna) Ministries of Health to implement a participatory action research cycle to generate innovative approaches for community engagement with the NTD programme, to ensure a detailed understanding of resources required for sustainable programme delivery. This will include a review of the direct and opportunity costs in relation to NTD treatment seeking and provision at the individual and household levels, as well as amongst programme implementers including community directed distributors (CDDs) and frontline health implementers.

\section{Measures of success in improving health}

There has been progress in establishing NTDs as a justifiable cause for investment, initiating action-oriented partnerships and establishing a respected 'brand' (Molyneux, 2012) with which policy makers, drug donors and philanthropists were prepared to align. This has provided an enabling environment to progress towards the targets established in the WHO Roadmap (WHO, 2012) and drive towards more equitable health interventions. Efforts have included scientific progress, sustained and high-level advocacy, commitment by the philanthropic community and NGDOs to the cause, which embraced not only health but also poverty alleviation as a development target (Hotez et al. 2009; Molyneux et al. 2017; Bangert et al. 2017). This has been enhanced by a consistent message around the role NTDs play in exacerbating poverty and the value for money provided by the interventions, some of which are dependent on donated high- 
quality assured products with delivery mechanisms able to reach those beyond 'the end of the road', often through sustained community commitment. The endorsement by the UN system and its member states following the inclusion of NTDs within the overall health targets of the SGDs has emphasized their importance as impediments to development. This has been highlighted by a detailed analysis of the impact that NTDs have when the other SDG targets are considered - improved access to water and sanitation, the overarching goal of poverty alleviation, improved food security and hunger alleviation, women's empowerment, improved education, absence of discrimination in disability and strengthened partnerships - all interplay with the broad NTD agenda, thereby contributing to the overarching goal of poverty alleviation (Hotez et al. 2009; Bangert et al. 2017).

The NTD community has also embraced a series of targets for elimination and control as well as a commitment to the eradication of Guinea Worm (dracunculiasis) and yaws following the publication of the WHO Roadmap in 2012. In 2013, the World Health Assembly (2013) endorsed the first comprehensive NTD Resolution on NTDs which outlined the five key strategies required for progress (WHA 66.12) including defining the milestones, linking NTDs to universal health coverage (UHC) and estimating the costs of NTD programmes through a detailed investment case (WHO, 2015c). The progress towards these targets is monitored annually for 10 NTDs through the role of Uniting to Combat NTDs (www.unitingtocombatntds.org). Details of country progress are regularly updated by WHO through reports in the WHO Weekly Epidemiological Record including country by country progress on annual treatments (WHO). NTDs are markers of poverty in many settings, including in poor areas and communities of G20 countries which together with Nigeria, have the highest number of people afflicted by NTDs (Hotez, 2017). Hence progress in their control, elimination or eradication will be a 'litmus test' of progress in reducing poverty. NTDs as 'tracers of equity' can provide an objective measure of overall progress to SDG goals (Engels, 2016) and thus implementing NTD strategies is an essential element of $\mathrm{UHC}$ to 'leave no one behind.

Making NTDs part of the UHC framing and a key indicator for SDGs is important; but success means turning rhetoric into action. Such realization is likely to involve the use of equity frameworks in programme planning and evaluation and participatory approaches to programme design and appraisal. These approaches require disaggregation of data by key equity markers such as gender, age, poverty and (dis)ability at all levels of the health system and critically using this data to ensure more responsive adaptation of planning. Whilst numbers are useful for identifying bottlenecks to equity, there is also a need to understand the lived experiences and challenges that affected individuals, their families, and frontline health providers (such as community-based drug distributors) face. An example where progress is being made is through the development of WHO's Gender Equity and Rights (GER) toolkit. This toolkit focuses on embedding GER analysis within routine monitoring and evaluation activities conducted by an NTD programme. The process uses routinely collected numerical data around NTD treatment/service provision combined with other equity indicators such as literacy rates or gender parity indicators to identify locations, where programme inequities may exist. More detailed, numerical analysis of indicators is then completed in these areas, before being complemented with a qualitative exploration of implementer (NTD programme staff, community drug distributors, frontline health facility staff) and community experience and perceptions of the NTD programme. Cumulative analysis of numerical data and data that captures the voices of affected populations, is then utilized to develop alternative steps in programme implementation that will increase programme equity in these areas thus creating an ongoing participatory cycle.

\section{Harnessing new technologies}

The opportunities to advance the NTD cause through the application of new technologies has been demonstrated by projects which have embraced the rapid expansion of cell phone networks and the reduced costs and availability of smartphones. This is perhaps best exemplified by the Global Trachoma Mapping Project (GTMP) which has enabled most rapid up-to-date information regarding the prevalence and distribution of trachoma globally. Late 2012 to early 2016 saw surveyors collect and transmit data from 2.6 million people in 29 countries using Android smartphones. The GTMP recorded on the International Trachoma Initiative database more districts in 3 years than had been recorded in the previous 12 years, and mapped areas where no data previously existed because of remoteness, insecurity, insufficient funding or competing public health priorities (www.sightsavers.org/gtmp/).

A new approach to detecting patients requiring care for lymphatic filariasis has been developed using the mHealth tool 'MeasureSMS-Morbidity' which allows health workers to use cell phones to record and transmit clinical information using short message services (SMS) (Mableson et al. 2017) for door-to-door surveys to obtain data on lymphatic filariasis patients in real-time (location, sex, age, clinical condition) in Dar-es-Salaam, Tanzania. This enabled identification, recording and mapping of lymphatic filariasis patients increases the efficiency of planning appropriate morbidity management and disability prevention (MMDP) activities (Mwingira et al. 2017). Similarly, the use of global positioning system data loggers is likely to become increasingly important to understand the movement of individuals and the dynamics of communities and individual behaviours and consequent transmission ecology (Brant et al. 2018).

Over the last decade the use of remote sensing and satellite imagery for defining habitat and broader ecological associations (Kelly-Hope et al. 2012; Thomson et al. 2002; Brito et al. 2017) projecting areas of transmission risk, and the need for a micromapping overlap stratification approach in Loa endemic areas, has enabled a better understanding of the interactions of ecology and epidemiology. Given the widespread availability of datasets and images of potential importance to the changing disease epidemiology and infection dynamics (forest and land cover, rainfall, altitude, geology), high-resolution imaging methodology is likely to be a more cost-effective approach to mapping and planning than ground truthing, given the rapidly changing ecology which drives the dynamics of transmission of vector-borne infection.

The application of network theory has also been used to ensure that there is a better understanding of the social behaviour of communities to enhance the efficiency of MDA (Chami et al. $2013,2016,2017)$. These approaches will enhance the efficiency of delivery of MDA and increase and sustain higher levels of adherence. A further potential approach that could be used for mapping, drug delivery and surveillance is the use of unmanned aerial vehicles (UAVs) or 'drones' (Fornace et al. 2014). UAVs can collect spatial data and their use in ecological research suggests they have potential in infectious disease epidemiology and public health research to provide spatial and temporal data in real-time, enabling an improved understanding of the interactions between disease transmission, vector ecology and environment.

\section{Capacity strengthening}

Significant progress on NTD capacity strengthening has been made. With the initial focus to support countries with the largest burdens of NTDs, a strategy which has ensured some progress towards increasing treatments in high burden countries (Nigeria, Ethiopia, Democratic Republic of the Congo, Tanzania and 
Table 1. The challenges of capacity strengthening

- Decentralizing learning activities from WHO to national institutions to facilitate the process of country adaptation, thus requiring the responsibility for activities such as quality assurance of laboratories, training courses, disseminating WHO materials and guidelines, ensuring timely drug applications and strengthening the supply chain.

- Countries should assume an advocacy function to ensure NTDs are central to any health training compatible with regional and specific country needs, and NTD activities need to be routinely embedded in health service personnel training. Information on new technical developments should be incorporated into national programmes within timeframes compatible with WHO targets.

- Monitoring, evaluation and surveillance towards verification of elimination are supported by quality assured laboratories and that resources are available for drug efficacy monitoring, entomological evaluation, safety monitoring, data management services, morbidity management and surgery.

- Training material should benefit from the most efficient means of dissemination using Apps and internet resources and m-health platforms.

- Sub-district and lower levels of the health system need to ensure that there is limited attrition of health staff and community-based health workers to maintain implementation.

- Engagement with the livestock sector to evaluate the most appropriate approaches to addressing zoonotic NTDs (zNTD) and elaborate the necessary partners to execute zNTD policies and strategies (WHO, 2015a).

- Recognize the challenges of training for intensified disease management where specific treatment regimens change as new drugs and diagnostics become available ensuring countries embrace new treatment policies and implement them at the earliest opportunity.

- Ensure that vector control and management are fully engaged, recognizing the particular challenges that countries face with a deficit in requisite skills in medical entomology, pesticide management, monitoring of insecticide resistance where insecticide resistance poses an ever-increasing threat and new products and novel approaches need to be introduced.

- Investigate the links and opportunities to engage with the WASH sector to disseminate materials pertinent to NTDs within WASH training programmes.

- Evaluate the curricula in all health training facilities (including medical schools) to ensure the recognition of NTDs as relevant topics for future health professionals.

- Support NTD programmes to utilize equity frameworks within routine health systems monitoring activities to allow for adaptation of programme delivery that is shaped by the needs of NTD community implementers and affected populations.

- Recognize that capacity strengthening activities can be mutually enhancing and that skills can be developed, learned and applied in the global north as well as in the global south.

Indonesia). Learning materials have been developed and training courses for national programme managers and district-level teams have been delivered. At Regional WHO levels, significant progress has been achieved though region specific or topic-specific meetings to advocate for a national commitment to NTD capacity development. Building capacity in research as well as in programme implementation has also been essential, whilst the African Research Network for NTDS (www.arntd.org) is a partnership model for research and a critical step forward in meeting this need. We summarize the needs for capacity strengthening in Table 1.

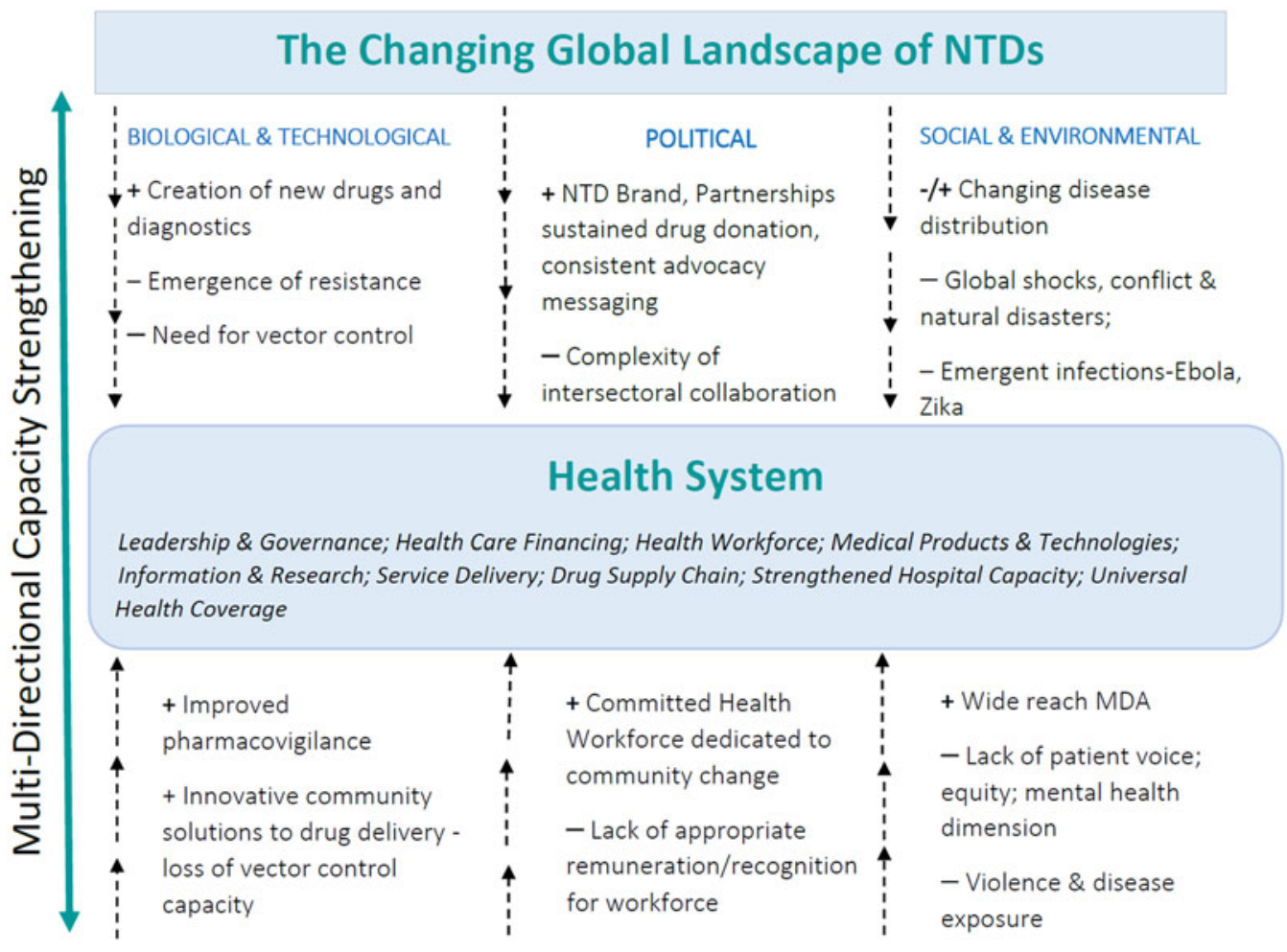

Communities

Fig. 1. The Health System as a mediator between the changing global landscape and the community: Moving towards responsive and resilient health systems to reach the NTD 'end game'. 


\section{Conclusion}

This paper has highlighted the challenges faced by the NTD community in achieving established targets in an ever-changing global world. Challenges that cut across the themes presented in this paper can be grouped into the biological and technological (e.g. the demand for new drugs, diagnostics and alternative vector control); political (e.g. ensuring prioritization of new and old challenges) and social and environmental (e.g. responding to global shocks and thinking beyond the parasite) (Bockarie et al. 2013; Webster et al. 2014) as shown in Fig. 1, spanning both the global and local levels.

Health systems ultimately become the mediator between the changing NTD global landscape and the realities of providing health care to poor communities. Multi-directional capacity strengthening, therefore, becomes critical in ensuring progress. As argued, this involves strategic partnership at different stages of the translational research continuum, a prioritization of equity facilitated through ongoing political commitments through the SDGs, and harnessing new technologies. Ultimately equitable partnerships for progress facilitated through multi-directional capacity strengthening are required to both build responsive and resilient health systems and to reach the NTD 'endgame'.

Financial support. The study was supported in part from the COUNTDOWN programme (grant ID PO 6407), a multi-disciplinary research consortium dedicated to investigating cost-effective, scaled-up and sustainable solutions, necessary to control and eliminate the seven most common NTDs by 2020. COUNTDOWN was formed in 2014 and is funded by UKAID part of the Department for International Development (DFID). The funders played no role in the decision to publish this article.

Conflict of Interest. DHM acknowledges support from GlaxoSmithKline. Other authors declare they have no Conflict of Interest.

\section{References}

African Research Network for NTDS (www.arntd.org).

Aljayyoussi G, Tyrer L, Ford L, Sjoberg H, Pionnier N, Waterhouse D, Davies J, Gamble J, Metugene H, Cook DA, Steven A, Sharma R, Guimaraes AF, Clare RH, Cassidy A, Johnston KL, Myhill L, Hayward L, Wanji S, Turner JD, Taylor MJ and Ward SA (2017) Short-Course, high-dose rifampicin achieves wolbachia depletion predictive of curative outcomes in preclinical models of lymphatic filariasis and onchocerciasis. Scientific Reports 7, 210.

Bailey F, Mondragon-Shem K and Hotez P (2017) A new perspective on cutaneous leishmaniasis-implications for global prevalence and burden of disease estimates. PLOS Neglected Tropical Diseases 11(8), e0005739. https://doi.org/10.1371/journal.pntd.0005739.

Bangert M, Molyneux D, H Lindsay SW, Fitzpatrick C and Engels D (2017) The cross-cutting contribution of the end of neglected tropical diseases to the sustainable development goals Infectious Diseases of Poverty 6, 73.

Bhatt S, Weiss DJ and Cameron E (2015) The effect of malaria control on Plasmodium falciparum in Africa between 2000 and 2015. Nature 526 (7572), 207-211.

Blackburn BG, Eigege A and Gotau H (2006) Successful integration of insecticide-treated bed net distribution with mass drug administration in Central Nigeria. American Journal of Tropical Medicine and Hygiene 75, 650-655.

Bockarie M, Kelly-Hope LH, Rebollo M and Molyneux DH (2013) Preventive chemotherapy as a strategy for elimination of neglected tropical parasitic diseases: endgame challenges. Philosophical Transactions of the Royal Society 368(20120144), DOI: 10.1098/rstb.2012.0144.

Bogus J, Gankpala L, Fischer K, Krentel A, Weil GJ, Fischer PU, Kollie K and Bolay FK (2016) Community attitudes toward mass drug administration for control and elimination of neglected tropical diseases after the 2014 outbreak of ebola virus disease in Lofa County, Liberia. American Journal of Tropical Medicine and Hygiene 94(3), 497-503.

Boussinesq M (2006) Loiasis. Annals of Tropical Medicine \& Parasitology 100, 715-731.
Brant TA, Okorie PN, Ogunmola O, Ojeyode NB, Fatunade SB, Davies E, Saka Y, Stanton MC, Molyneux DH, Russell Stothard J and KellyHope LA (2018) Integrated risk mapping and landscape characterisation of lymphatic filariasis and loiasis in South West Nigeria. Parasite Epidemiology and Control 3, 21-35. doi: https://doi.org/10.1016/j.parepi. 2017.12.001.

Brito M, Paulo R, Van-Dunem P, Martins A, Unnasch TR, Novak RJ, Jacob B, Stanton MC, Molyneux DH and Kelly-Hope LA (2017) Rapid integrated clinical survey to determine prevalence and co-distribution patterns of lymphatic filariasis and onchocerciasis in a Loa loa co-endemic area: The Angolan experience Parasite Epidemiology and Control 2, 71-84.

Chami GF, Molyneux DH, Kontoleon AA and Dunne DW (2013) Exploring network theory for mass drug administration. Trends in Parasitology 29, 370-379.

Chami GF, Kontoleon A, Bulte E, Fenwick A, Kabatereine NB, Tukahebwa EM and Dunne DW (2016) Profiling nonrecipients of mass drug administration for schistosomiasis and hookworm infections: a comprehensive analysis of praziquantel and albendazole coverage in communitydirected treatment in Uganda. Clinical Infectious Diseases 62, 200-207.

Chami GF, Ahnert SE, Kabatereine NB and Tukahebwa EM (2017) Social network fragmentation and community health. Proceedings of the National Academy of Sciences of the United States of America 10.1073.

Coleman M, Foster GM, Deb R, Pratap Singh, Smail HM, Shivam P, Ghosh AK, Dunkley S, Kumar V, Coleman M, Hemingway J, Paine MJ and Das P (2015) DDT-based indoor residual spraying suboptimal for visceral leishmaniasis elimination in India. Proceedings of the National Academy of Sciences of the United States of America 112, 8573-8578.

COR-NTD (www.ntdsupport.org/cor-ntd)

Dean L, Page S, Hawkins K, Stothard R, Thomson R, Wanji S, Gyapong M, Anagbogu I, Molyneux D and Theobald S (2016) Tailoring mass drug administration to context: implementation research is critical in achieving equitable progress in the control and elimination of helminth neglected tropical diseases in sub-Saharan Africa. International Health 8 , 233-234.

De Vlas SJ, Stolk WA, le Rutte EA, Hontelez JA, Bakker R, Blok DJ, Cai R, Houweling TA, Kulik MC, Lenk EJ, Luyendijk M, Matthijsse SM, Redekop WK, Wagenaar I, Jacobson J, Nagelkerke NJ and Richardus JH (2016) Concerted efforts to control or eliminate neglected tropical diseases: how much health will Be gained? PLoS Neglected Tropical Diseases 10(2), e0004386. doi: 10.1371/journal.pntd.0004386.

Dias JC, Silveira AC and Schofield CJ (2002) The impact of Chagas disease control in Latin America: a review. Memorias do Instituto Oswaldo Cruz 97, 603-612.

Drugs for Neglected Diseases initiative (DNDi) (www.dndi.org).

Eigege A, Kal E, Miri E, Sallau A, Umaru J, Mafuyai H, Chuwang YS, Danjuma G, Danboyi J, Adelamo SE, Mancha BS, Okoeguale B, Patterson AE, Rakers L and Richards FO (2013) Long-lasting insecticidal nets are synergistic with mass drug administration for interruption of lymphatic filariasis transmission in Nigeria. PLoS Neglected Tropical 7 (10), e2508.

Engels D (2016) Neglected tropical diseases in the sustainable development goals. Lancet 387, 223-234.

Ferrari AJ, Charlson FJ, Norman RE, Patten SB, Freedman G, Murray CJ, Vos T and Whiteford HA (2013) Burden of depressive disorders by country, sex, age and year:findings from the global Burden of Disease Study 2010. PLoS Medicine 10, e1001547.

Fornace KM, Drakeley CJ, William T, Espino F and Cox J (2014) Mapping infectious disease landscapes: unmanned aerial vehicles and epidemiology. Trends in Parasitology 30, 514-519.

Gardon J, Gardon-Wendel N, Demanga-Ngangue, Kamgno J, Chippaux JP and Boussinesq M (1997) Serious reactions after mass treatment of onchocerciasis with ivermectin in an area endemic for Loa loa infection. Lancet 350, 18-22.

Gilson L, Barasa E, Nxumalo N, Cleary S, Goudge J, Molyneux S, Tsofa B and Lehmann U (2017) Everyday resilience in district health systems: emerging insights from the front lines in Kenya and South Africa. BMJ Global Health 0.1136/bmjgh-2016-000224.

Hemingway J, Ranson H, Magill A, Kolaczinski J, Fornadel C, Gimnig J, Coetzee M, Simard F, Roch DK, Hinzoumbe CK, Pickett J, Schellenberg D, Gething P, Hoppé M and Hamon N (2016) Averting a malaria disaster: will insecticide resistance derail malaria control? Lancet S0140-6736(15)00417-1. 
Hodges ME, Koroma JB, Sonnie M, Kennedy N, Cotter E and Macarthur C (2011) Neglected tropical disease control in post-war Sierra Leone using the onchocerciasis control programme as a platform. International Health 3, 69-74.

Hollingsworth TD, Adams EA and Anderson RA and the NTD Modelling Consortium. (2015) Quantitative analyses and modelling to support achievement of the 2020 goals for nine neglected tropical diseases. Parasites \& Vectors 8, 630. doi: 10.1186/s13071-015-1235-1.

Hotez PJ, Alvarado M, Basáñez MG, Bolliger I, Bourne R, Boussinesq M, Brooker SJ, Brown AS, Buckle G, Budke CM, Carabin H, Coffeng LE, Fèvre EM, Fürst T, Halasa YA, Jasrasaria R, Johns NE, Keiser J, King CH, Lozano R, Murdoch ME, O'Hanlon S, Pion SD, Pullan RL, Ramaiah KD, Roberts T, Shepard DS, Smith JL, Stolk WA, Undurraga EA, Utzinger J, Wang M, Murray CJ and Naghavi M (2014) The global burden of disease study 2010: interpretation and implications for the neglected tropical diseases. PLoS Neglected Tropical Diseases 7, e2865.

Hotez PJ, Fenwick A, Savioli L and Molyneux DH (2009) Rescuing the bottom billion through control of neglected tropical diseases. Lancet 373, 1570-1575.

Hotez PJ (2017) Blue Marble Health. Baltimore, USA: Johns Hopkins University Press, 205p.

InfoNTD website (www.infontd.org).

Integrated Vector Control Consortium (IVCC) (www.ivcc.com)

Kelly-Hope LA, Bockarie MJ and Molyneux DH (2012) Loa loa ecology in Central Africa: role of the Congo river system. PLoS Neglected Tropical Diseases 6.0001605.

Kelly-Hope LA, Paulo R, Thomas B, Brito M, Unnasch TR and Molyneux D (2017) Loa loa vectors Chrysops spp.: perspectives on research, distribution, bionomics, and implications for elimination of lymphatic filariasis and onchocerciasis. Parasites \& Vectors 10, 172.

Liese BL, Houghton N and Teplitskaya L (2014) Development assistance for neglected tropical diseases: progress since. International Health 6, 162-171.

Liese B, Rosenberg M and Schratz P (2010) Programmes, partnerships, and governance for elimination and control of neglected tropical diseases. Lancet 375, 67-76.

Liese B and Schubert L (2009) Official development assistance for healthhow neglected are neglected tropical diseases? An analysis of health financing. International Health 1, 141-47.

Litt E, Baker M and Molyneux DH (2012) Neglected tropical diseases and mental health: a perspective on comorbidity. Trends in Parasitology 28, 195-201.

Mableson HE, Martindale S, Stanton MC, Mackenzie C and Kelly-Hope LA (2017) Community-based field implementation scenarios of a short message service reporting tool for lymphatic filariasis case estimates in Africa and Asia. Mhealth 3, 28.

Mieras LF, Anand S, van Brakel WH, Hamilton HC, Martin Kollmann KH, Mackenzie C, Mason I and Wickenden A (2016) Neglected tropical diseases, cross-cutting issues workshop, 4-6 February 2015, Utrecht, The Netherlands: meeting report. International Health 8(1), 7-11.

Molyneux DH (2012) The 'neglected tropical diseases': now a brand identity; responsibilities, context and promise. Parasites \& Vectors 30(5), 23.

Molyneux DH, Savioli L and Engels D (2017) Neglected tropical diseases: progress towards addressing the chronic pandemic. Lancet 389, 312-325.

Murray CJ, Vos T, Lozano R, Naghavi M, Flaxman AD, Michaud C, Ezzati M, Shibuya K, Salomon JA, Abdalla S, Aboyans V, Abraham J, Ackerman I, Aggarwal R, Ahn SY, Ali MK, Alvarado M, Anderson HR, Anderson LM, Andrews KG, Atkinson C, Baddour LM, Bahalim AN, Barker-Collo S, Barrero LH, Bartels DH, Basáñez MG, Baxter A, Bell ML, Benjamin EJ et al. (2012) Disability-adjusted life years (dalys) for 291 diseases and injuries in 21 regions, 1990-2010: a systematic analysis for the global burden of disease study. Lancet 9859, 2197-2223.

Mwingira U, Chikawe M, Mandara WL, Mableson HE, Uisso C, Mremi I, Malishee A, Malecela M, Mackenzie CD, Kelly-Hope LA and Stanton MC (2017) Lymphatic filariasis patient identification in a large urban area of Tanzania: an application of a community-led mHealth system. PLoS Neglected Tropical Diseases 11(7), e0005748.

NNN -NGDO/NTD/Network (www.ntd-ngonetwork.org)

Nsakashalo-Senkwe M, Mwase E, Chizema-Kawesha E, Mukonka V, Songolo P, Masaninga F, Rebollo MP, Thomas B, Bockarie MJ,
Betts H, Stothard JR and Kelly-Hope LA (2017) Significant decline in lymphatic filariasis associated with nationwide scale-up of insecticide-treated nets in Zambia. Parasite Epidemiology and Control 2, 4-17.

Pion SDS, Chesnais CB, Weil GJ, Fischer PU, Missamou F and Boussinesq M (2017) Effect of 3 years of biannual mass drug administration with albendazole on lymphatic filariasis and soil-transmitted helminth infections: a community-based study in Republic of the Congo. Lancet Infectious Diseases 17, 763-769.

Pose R and Rabinowitch G (2014) Overseas Development Institute Development progress. NO LONGER NEGLECTED Tackling Sierra Leone's neglected tropical diseases.

Rebollo MP, Sambou SM, Thomas B, Biritwum N-K, Jaye MC, KellyHope L, Escalada AG, Molyneux DH and Bockarie MJ (2015) Elimination of lymphatic filariasis in Gambia. PLoS Neglected Tropical Diseases doi: 10.1371/journal.pntd.0003642.

Reidpath DD, Allotey P and Pokhrel S (2011) Social science research in neglected tropical diseases 2: a bibliographic analysis. Health Research Policy and Systems 9, 1.

Sightsavers. Global Trachoma Mapping Project www.sightsavers.net/our work/how_we_work/.../18687.html.

Thomas BC, Kollie K, Koudou B and Mackenzie C (2017) Commentary: restarting NTD programme activities after the Ebola outbreak in Liberia. Infectious Diseases of Poverty 6(1), 52.

Thomson MC, Obsomer V, Dunne M, Connor SJ and Molyneux DH (2002) Satellite mapping of Loa loa prevalence in relation to ivermectin use in west and Central Africa. Lancet 356, 1077-1078.

Tirados I, Esterhuizen J, Kovacic V, Mangwiro TN, Vale GA, Hastings I, Solano P, Lehane MJ and Torr SJ (2015) Tsetse control and gambian sleeping sickness; implications for control strategy. PLoS Neglected Tropical Diseases 10.1371 .

Ton T, Mackenzie C and Molyneux DH (2015) The burden of mental health in lymphatic filariasis. Infectious Diseases of Poverty 30(4), 34.

Turner J, Ford D, Tyrer L, Sjoberg J, Gamble J, Cook D, von Geldern T, Marsh K, Wanji S, Ward S and Taylor MJ (2015) Tylosin analogs as antifilarial agents. Part B: pre-clinical evaluation in rodent models of lymphatic filariasis and onchocerciasis" Abstract 645 American Society of Tropical Medicine and Hygiene $64^{\text {th }}$ Annual Meeting Philadelphia USA.

Uniting to Combat NTDs (www.unitingtocombatntds.org).

Webber RH (1979) Eradication of Wuchereria bancrofti infection through vector control. Transactions of the Royal Society of Tropical Medicine and Hygiene 73, 722-724.

Webster JP, Molyneux DH, Hotez PJ and Fenwick A (2014) The contribution of mass drug administration to global health-past, present and future. Philosophical Transactions of the Royal Society B: Biological Sciences 12, 369.

World Health Assembly (2013) Neglected tropical diseases prevention, control, elimination and eradication. World Health Assembly 66, 12.

World Health Organization (2012) A Roadmap for Implementation: Accelerating work to overcome the global impact of neglected tropical diseases.

World Health Organization (2015a) The control of neglected zoonotic diseases: from advocacy to action. Report of the fourth international meeting held at WHO Headquarters, Geneva, Switzerland, 19-20 November 2014. 44 pages Editor B. Abela-Ridder; Geneva, Switzerland.

World Health Organization (2015b) Water Sanitation \& Hygiene for Accelerating and Sustaining Progress on Neglected Tropical Diseases-A Global Strategy 2015-2020. Geneva: World Health http://apps.who.int/iris/ bitstream/10665/182735/1/WHO_FWC_WSH_15.12_eng.pdf?ua=1.

World Health Organization (2015c) Investing to overcome the global impact of neglected tropical diseases: WHO third report on neglected tropical diseases: https://www.yumpu.com/en/document/view/37074971/1a9bnbk/5.

World Health Organization (2016) World Malaria Report. 186 pages. ISBN: 9789241511711 Geneva, Switzerland.

World Health Organization (2017a) Eliminate Yellow Fever Epidemics. http:// www.who.int/csr/disease/yellowfev/african-countries-endorse-eye-strategy/en/.

World Health Organization (2017b) Global Vector Control Response 2017-2030 Draft www.who.int/entity/vector-control/publications/globalcontrol-response/en/ - 30k. 